\title{
Management of Fluid Overload in the Pediatric ICU
}

\author{
Grace L. Ker and Sandeep Gangadharan
}

\section{Introduction}

The clinical implications of fluid overload and its management currently remain controversial within the pediatric critical care literature. Resuscitation with intravenous fluids is often necessary in critical care for maintenance of perfusion during shock or hypovolemic states. In recent years, a shift toward early recognition of sepsis has encouraged rapid and early initiation of fluid at illness presentation with the hopes of improving outcomes. As a consequence, aggressive early fluid resuscitation can contribute to fluid overload states in the setting of critical illness. Recent literature in both adult and pediatric patients suggests that fluid overload in critical illness can have harmful effects and contribute to morbidity and mortality [1]. However, the definition and management of fluid overload remain unclear, likely reflecting the complex relationship between volume resuscitation, fluid distribution, and critical illness.

\footnotetext{
G. L. Ker $(\bowtie) \cdot$ S. Gangadharan

Department of Pediatric Critical Care, Cohen

Children's Medical Center,

New Hyde Park, NY, USA
}

\section{Pathophysiology}

Some of the difficulties surrounding fluid management in critically ill patients arise from our incomplete understanding of the movement and distribution of fluid within the body, particularly in the setting of systemic disease states and therapeutic agents that impact endothelial permeability and vascular autoregulation.

Total body water accounts for approximately $60 \%$ of body weight and is distributed between the intracellular space (ICS) and the extracellular space (ECS). The percentage of water per body weight fluctuates with age, such that premature infants and term newborns have higher total body water relative to adults, which rapidly decreases to adult values during the first year of life [2]. The intracellular space accounts for $55 \%$ of total body water, while the remaining $45 \%$ exists within the ECS. The ECS is further divided into three compartments: intravascular space or plasma (IVS), interstitial space (ISS), and transcellular space (TCS), which account for $15 \%, 45 \%$, and $40 \%$, respectively, of total extracellular fluid. The transcellular space refers to digestive, cerebrospinal, intraocular, pleural, peritoneal, and synovial fluids [3]. The movement of water between the three compartments of the extracellular space occurs through a semipermeable membrane [4].

The homeostatic state of fluid movement within the body is determined by a balance between the physical properties between the 
fluids that exist within each individual compartment and the properties of the semipermeable membranes that separate them, as detailed in Table 11.1 [4]. Fluid movement is further tightly regulated by feedback loops involving the central nervous, endocrine, and renal systems [2].

Movement of fluids across the capillary bed had previously been largely described through the Starling principle, which relies upon assumptions that the capillary and postcapillary venules behave as semipermeable membranes absorbing fluid from the interstitial space. This relationship is illustrated through the use of Eq. 11.1. In this assumption, transvascular exchange depends largely upon a gradient between oncotic and hydrostatic pressure. At the arterial end, the dominant pressure gradient present is that of a hydrostatic pressure differential, and therefore, the driving force for fluid movement is away from the higher capillary hydrostatic pressure and

Table 11.1 Summary of properties of body fluids by compartment

\begin{tabular}{l|l|l|l}
\hline Properties & $\begin{array}{l}\text { Plasma } \\
\text { or IVS }\end{array}$ & $\begin{array}{l}\text { Interstitial } \\
\text { fluid }\end{array}$ & $\begin{array}{l}\text { Intracellular } \\
\text { fluid }\end{array}$ \\
\hline $\begin{array}{l}\text { Colloid osmotic } \\
\text { pressure }(\mathrm{mmHg})\end{array}$ & 25 & 4 & 0 \\
\hline $\begin{array}{l}\text { Osmolality } \\
(\mathrm{mOsm} / \mathrm{kg})\end{array}$ & 280 & 280 & 280 \\
\hline $\mathrm{pH}$ & 7.4 & 7.4 & 7.2 \\
\hline $\mathrm{Na}+(\mathrm{mmol} / \mathrm{L})$ & 142 & 143 & 10 \\
\hline $\mathrm{K}+(\mathrm{mmol} / \mathrm{L})$ & 4 & 4 & 155 \\
\hline $\mathrm{Cl}-(\mathrm{mmol} / \mathrm{L})$ & 103 & 115 & 8 \\
\hline $\mathrm{Ca}++(\mathrm{mmol} / \mathrm{L})$ & 2.5 & 1.3 & $<0.001$ \\
\hline
\end{tabular}

Table created by Grace Ker toward the lower interstitial fluid hydrostatic pressure. Fluid movement in this direction is termed filtration (Fig. 11.1) [4].

Starling's Equation for Fluid Movement

$$
\mathrm{JV}=\mathrm{Kf}\left[\left(\mathrm{P}_{\mathrm{c}}-\mathrm{P}_{\mathrm{is}}\right)-\sigma\left(\pi_{\mathrm{c}}-\pi_{\mathrm{is}}\right)\right]
$$

$\mathrm{Kf}=$ Filtration constant

$\mathrm{JV}=$ Fluid movement

$\mathrm{Pc}=$ Capillary pressure

Pis= Interstitial fluid pressure

$\pi_{\mathrm{c}=}$ Capillary colloid osmotic pressure

$\pi_{\text {is }}=$ Interstitial colloid osmotic pressure

As fluid moves throughout the length of the capillary bed toward the venule end, filtration will eventually lead to a comparatively increased concentration of proteins within the intravascular space and thus an increased intravascular oncotic pressure in comparison to the interstitial fluid. This differential in oncotic pressure at the venule end of the capillary is greater than the hydrostatic differential, and so there is reversal of flow based on an overall pressure gradient that leads to absorption of fluid back into the intravascular space [4]. This process is summarized in Fig. 11.2. The Starling concept of filtration and absorption for fluid movement is demonstrated in Fig. 11.3.

Despite Starling's principles of fluid movement being generally considered the standard, in 2004, Adamson and colleagues revised the Starling equation, theorizing that non-fenestrated capillaries filter fluid throughout their entire length into the interstitial space and that absorption through the venules does not occur despite a rise in capillary oncotic pressure, which is unable
Fig. 11.1 Diagram demonstrating relationship between hydrostatic pressures within the capillary bed at the arterial end. (Image created by Grace Ker)

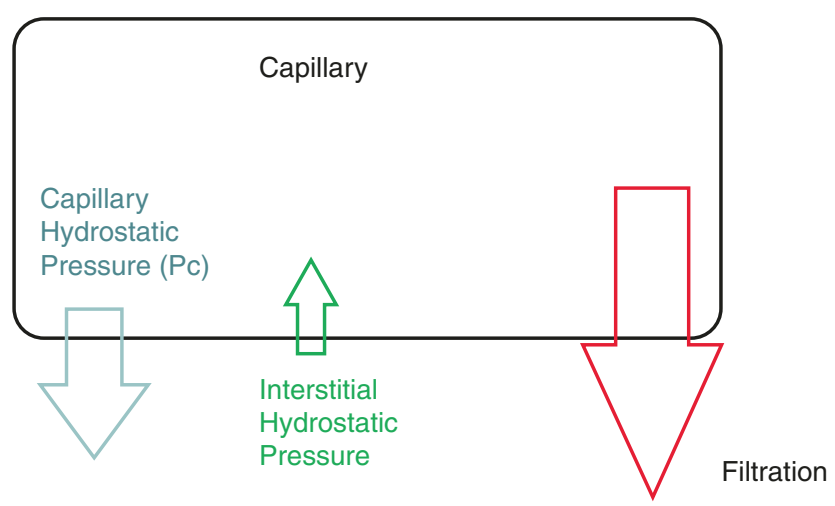


Fig. 11.2 Diagram demonstrating relationship between oncotic pressures within the capillary bed at the venule end. (Image created by Grace Ker)
Venule End

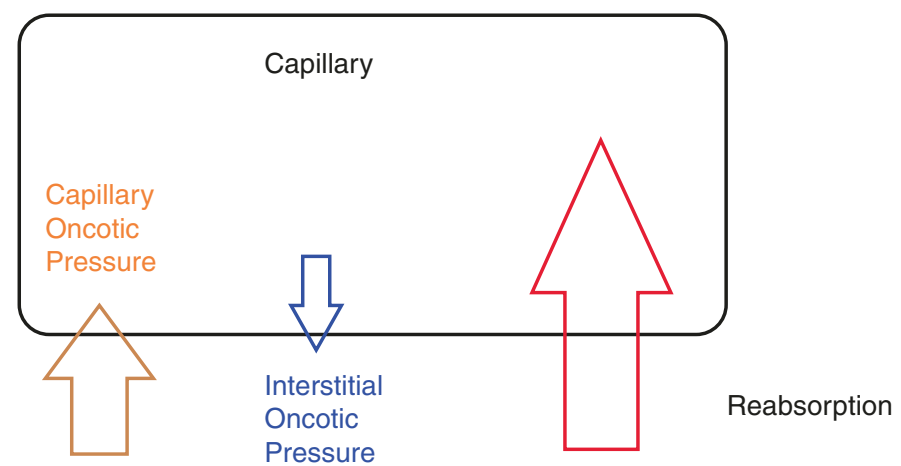

\section{THE CLASSICAL MODEL OF THE STARLING PRINCIPLE}

In the precapillary arterioles, the hydrostatic pressure is greater than the oncotic pressure, favouring the movement of water into the interstitial fluid
In the postcapillary venules, the oncotic pressure is greater than the hydrostatic pressure, favouring the movement of water out of the interstitial fluid and back into the venules

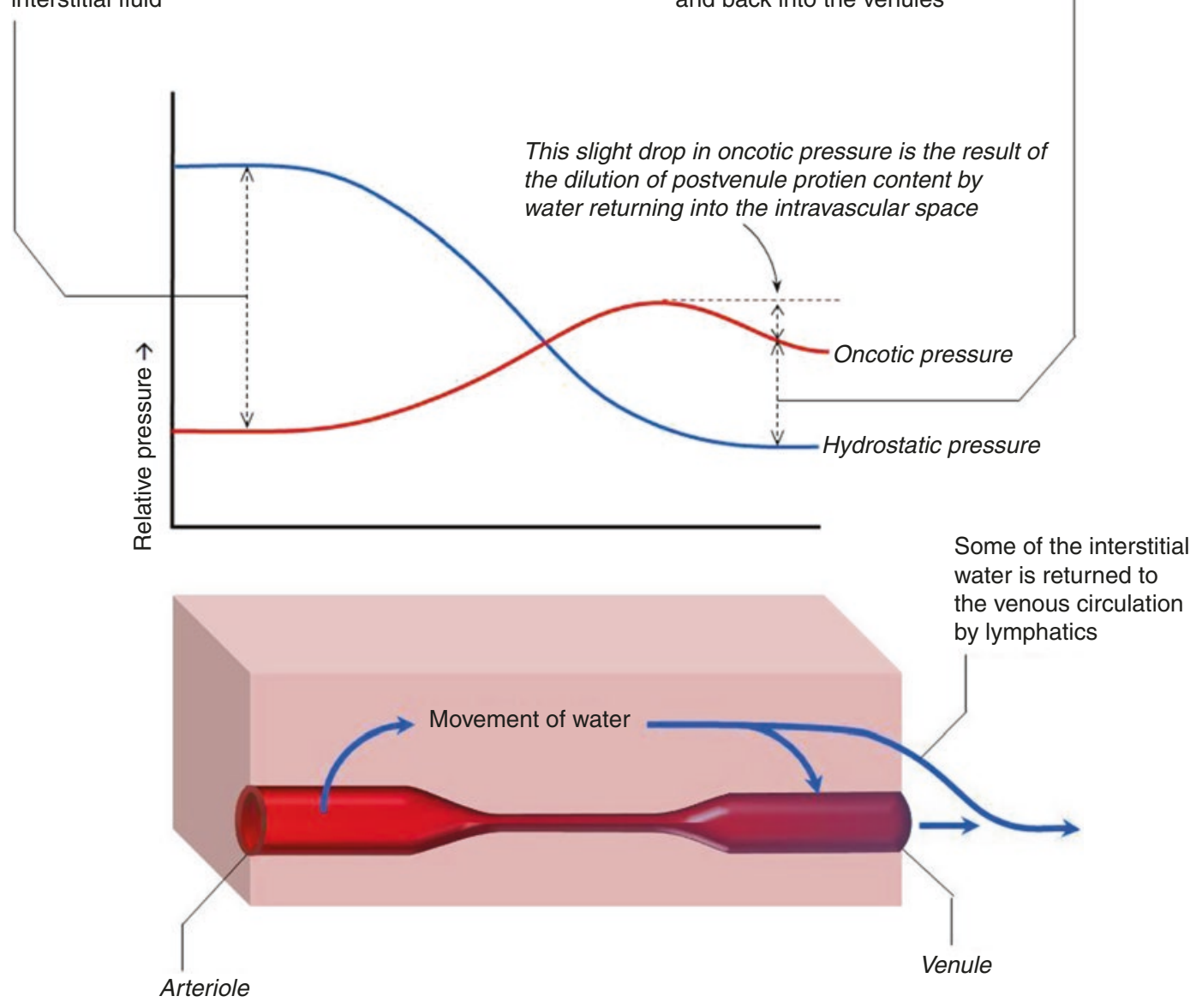

Fig. 11.3 Image demonstrating the Starling principle for fluid movement. At the arterial end, hydrostatic pressure exceeds oncotic pressure with relative movement of water into the interstitial fluid via filtration. At the venule end, oncotic pressure is greater than hydrostatic pressure which then favors the movement of fluid into the vasculature via absorption. ("Image courtesy of Alex Yartsev, www.derangedphysiology.com") 
to overcome hydrostatic forces. Therefore, there is no true reversal of filtration. Instead, filtered fluid circulates throughout the interstitial space and largely returns to the circulation as lymph [5]. This principle for fluid movement is influenced by substances that exist in the multiple layers between the interstitial space and intravascular space [5]. These layers include a subglycocalyx space which is largely protein-free and an endothelial glycocalyx space which consists of a matrix of glycoproteins and proteoglycans. The endothelial glycocalyx layer serves as a semipermeable membrane separating plasma from interstitial space. Rather than gradients between the interstitial oncotic pressure and intravascular oncotic pressure that serve as a driving force for fluid movement, it is the subglycocalyx capillary oncotic pressure $(\pi \mathrm{s})$ that serves as the major determinant in transcapillary flow ( Jv) [5]. This model and the function of the endothelial glycocalyx in fluid movement are illustrated in Fig. 11.4. Further research into the translation of

Part 1

\section{THE ROLE OF THE GLYCOCALYX IN TRANSVASCULAR FLUID EXCHANGE}

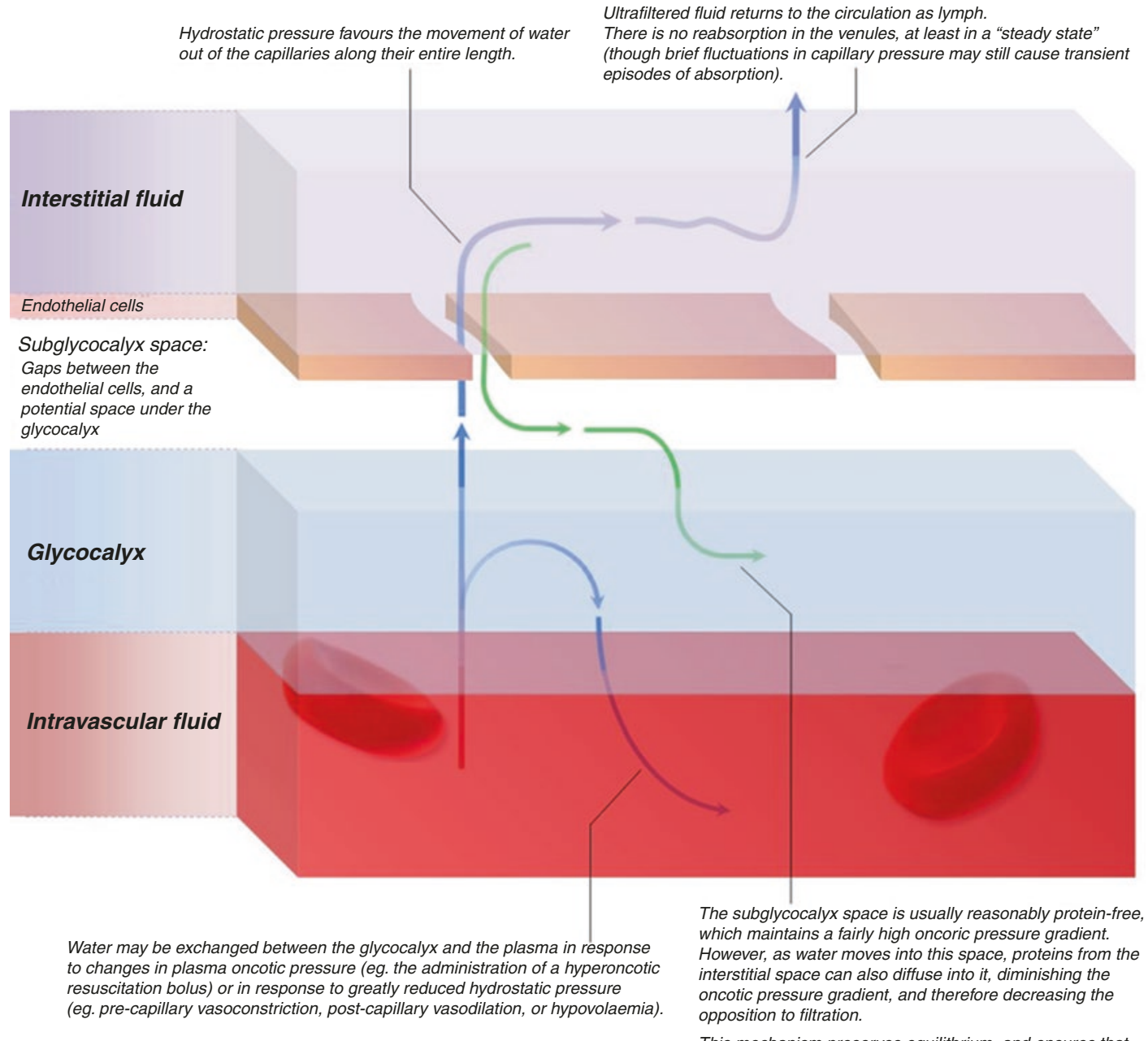

Fig. 11.4 Image demonstrating the role of the endothelial glycocalyx in fluid exchange (part 1) and revised model of the Starling principle (part 2) involving the subglycocalyx illustrating the increase in oncotic pressure throughout the vessel does not exceed hydrostatic pressure with no true reversal of filtration occurring. ("Image courtesy of Alex Yartsev, www.derangedphysiology. com") 
Part 2

\section{THE REVISED MODEL OF THE STARLING PRINCIPLE} In the precapillary arterioles, the hydrostatic pressure
is greater than the oncotic pressure, favouring the
movement of water into the subglycocalyx space and
thus into the interstitial fluid.
In the postcapillary venules, the hydrostatic pressure has decreased, but the oncotic pressure still only opposes (and does not reverse) the movement of fluid. The magnitude of this opposition is somewhat diminished by the influx of protein into the subglycocalyx.

Thus, the net direction of fluid movement is still out of the venules.
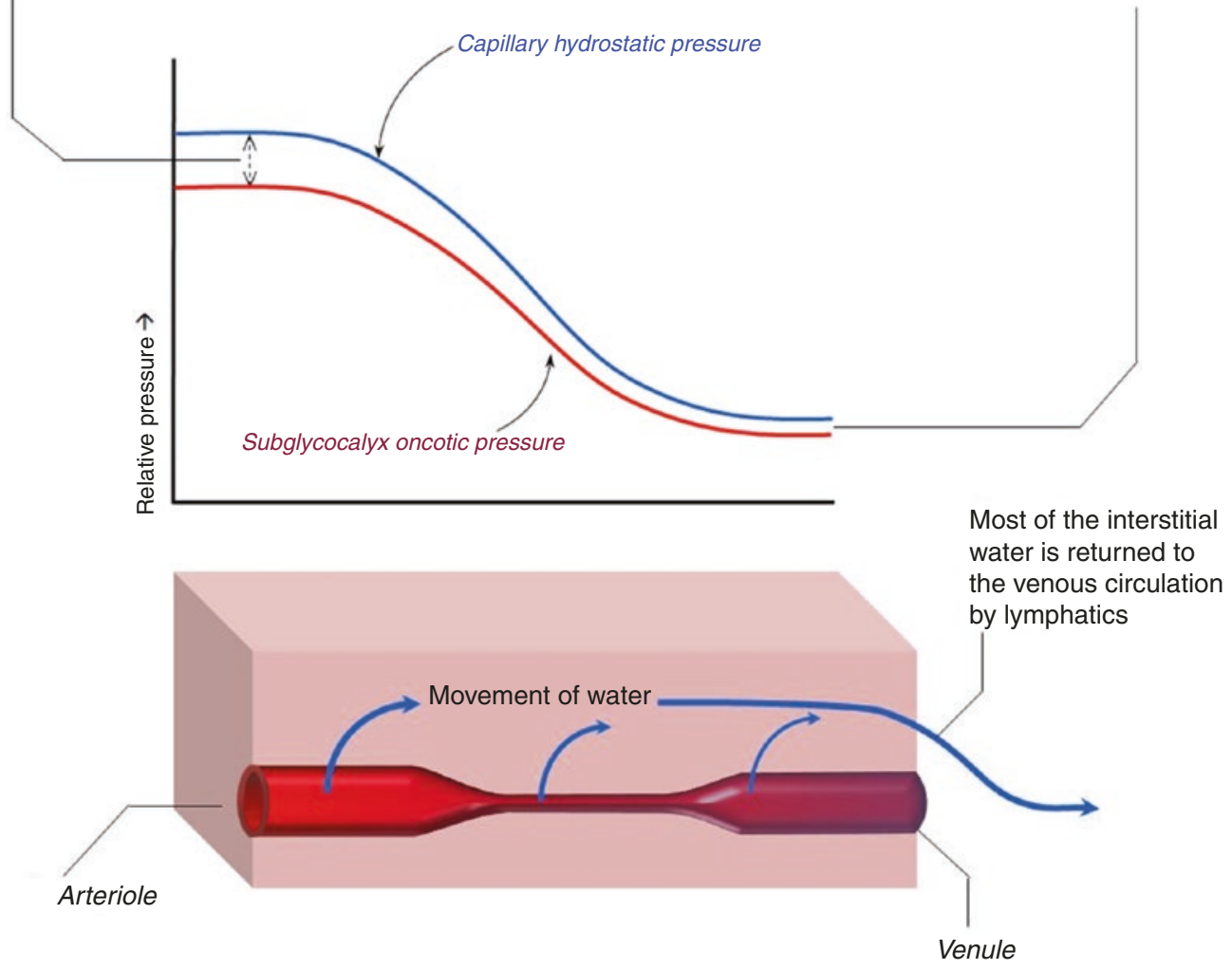

Fig. 11.4 (continued)

this model into clinical practice could lead to improvements in the management of fluid status in critically ill patients.

Fluid management strategies, particularly in states of shock, are often complicated by the need to balance maintenance of oxygen delivery to match metabolic demand and maintain cardiac output without avoiding fluid overload. Shock states and critical illness often have underlying systemic inflammation resulting in anasarca and capillary leak, altering normal fluid movement between compartments [6]. Dysregulation of fluid distribution within intracellular and extracellular compartments, along with aggressive fluid resuscitation and impaired renal clearance, ultimately leads to hypervolemia and fluid overload.

Fluid overload states can impact organ function influencing morbidity and mortality. Excessive fluid can alter the efficiency of the heart by moving ventricular compliance far to the right on the Frank-Starling curve, potentially resulting in impaired cardiac function. Hypervolemia can cause pulmonary alveolar and interstitial pulmonary edema, precipitating respiratory failure [7]. Specifically, worsening pulmonary edema will negatively affect lung compliance, potentiate V/Q mismatch, and, consequently, worsen both ventilation and oxygenation. Finally, particularly during stress states, 
renal perfusion may be impaired causing the development of acute kidney injury (AKI), which further complicates the ability of kidneys to maintain euvolemia.

An understanding of fluid distribution and movement in the body therefore becomes critical during periods of fluid resuscitation and overload. Management strategies targeting such pathophysiologic mechanisms are designed to help reduce morbidity and mortality associated with fluid overload and multiorgan dysfunction.

\section{Measurement of Fluid Overload}

\section{Case}

A 12-year-old female presents to the emergency room with septic shock in the setting of acute influenza infection requiring intubation and aggressive fluid resuscitation. Four days after presentation, her hemodynamics have improved, and she is able to be weaned off inotropic support without difficulty. Her chest X-ray is significant for pulmonary edema. A central venous catheter within the internal jugular vein measures a central venous pressure of 5 $\mathrm{CmH}_{2} \mathrm{O}$. Her weight is $2 \mathrm{~kg}$ above her baseline, but her intakeloutput flowsheet shows that she is overall in a negative fluid balance of $1 \mathrm{Lfor}$ the duration of her hospitalization. Among the physician team caring for her, there is disagreement about her hydration status. Some physicians feel that she is euvolemic, while others feel that she is grossly fluid overloaded. Possible management pathways are discussed at length. A nurse asks if there is one clear best method to evaluate for fluid overload in a critically ill pediatric patient.

Despite an acknowledgement that fluid overload can be associated with poor outcomes in critical care populations, the optimal clinical definition of fluid overload remains unclear [8]. In
2001, Goldstein et al. developed definitions for fluid overload that relied upon measuring fluid input and output from PICU admission [9]. This method for calculation of fluid overload is demonstrated below:

$$
\% \mathrm{FO}=\frac{\begin{array}{l}
\text { Sum of daily fluid } \\
\text { (fluid in }- \text { fluid out })
\end{array}}{\text { ICU admission weight }} \times 100 \text {. }
$$

The majority of pediatric studies since then have largely utilized this definition for the purposes of research and clinical identification. However, this method is potentially error prone as it relies upon precise accounting of daily fluid balance and accurate calculations. As an alternative method, fluctuations in weight have been used in some studies. For example, in a study of patients receiving continuous renal replacement therapy (CRRT), measurements of weight at hospital or ICU admission and initiation of CRRT were utilized to determine fluid overload percentage with formulas as demonstrated below [8]:

$$
\% \mathrm{FO}=\frac{\begin{array}{c}
\text { CRRT initiation weight }- \text { ICU } \\
\text { admission weight }
\end{array}}{\text { ICU admission weight }} \times 100
$$

OR

$$
\% \mathrm{FO}=\frac{\begin{array}{c}
\text { CRRT initiation weight }- \text { Hospital } \\
\text { admission weight }
\end{array}}{\text { Hospital admission weight }} \times 100 \text {. }
$$

These calculations were compared with the standard method of intake and output assessment and were found to have a high degree of correlation with similar predictive ability for mortality [8]. Therefore, it is possible the two methods could be utilized interchangeably in the clinical setting, though the method of utilizing daily weights rather than summation of daily fluid intake is likely the less labor-intensive means of determining fluid overload.

Despite the fact that both calculations have been validated and are relatively easy to perform, discrepancies between weight measurements and intake/output calculations often occur, thereby complicating management decisions. These discrepancies likely result from the limitations of 
these two methods. For instance, fluid-based calculations are unable to fully account for insensible losses, and weight-based calculations are vulnerable to errors that can occur through the use of different scales or weighing techniques. One relatively novel method devised to address such issues involves the use of bioimpedance as a measurement tool to determine fluid overload status.

Bioimpedance utilizes electrical properties of biological tissues in evaluating their response to electrical current with either active (in which electrostimulation triggers ionic activities inherent within tissue cells) or passive response (i.e., response only occurs with external stimulation) [10]. The ability of tissues to impede frequency signals is then extrapolated mathematically to calculate the composition of the tissue. While it has a variety of medical applications, bioimpedance has been proposed as a method to evaluate for fluid status due to its ability to estimate total body fluid and extracellular fluid. However, although multifrequency bioimpedance has been reported as a reliable measure of estimating body water compartments in adults [10], the pediatric literature to support its use remains sparse. Milani et al. recently attempted to evaluate the use of multifrequency bioimpedance measurements in pediatric patients requiring maintenance dialysis and found that measurements of body water compartments were imprecise when compared with gold standard measurements of bromide or deuterated water dilution, two methods which, while accurate, remain impractical for daily clinical use [10]. Although bioimpedance may serve as a potential noninvasive alternative to assess for fluid status in the future, its reliability in critically ill pediatric populations has yet to be realized.

The determination of fluid overload in the critical care setting depends upon the accurate assessment of intake and output from admission and relating this information to each patient's baseline weight. Our recommendation is to use the Goldstein method of daily intake and output in comparison from admission weight to evaluate for percentage of fluid overload [9]. When fluid intake or output measurements are unavailable or incomplete, daily weight measurements can be substituted as a reliable method to measure fluid overload. Regardless of the method of fluid overload measurement, interpretation of fluid status still requires clinician expertise, particularly in settings where physical exam and calculated values are discrepant. The child in the scenario above experienced a significant inflammatory response and capillary leak secondary to her infectious process which predisposed her to pulmonary edema and fluid overload. It is not uncommon for critically ill patients to have intravascular volume depletion concurrent with total body fluid overload. Therefore, despite signs of fluid overload on clinical examination, if the patient were to remain hypotensive or unable to tolerate diuresis, it would be suggestive of ongoing low intravascular volume despite overall fluid overload which would alter clinical decision-making regarding the timing of diuresis or fluid removal therapies.

\section{Managing Fluid Overload with Renal Replacement Versus Diuretic Therapy}

\section{Case}

A 16-year-old female presents with septic shock in the setting of Staphylococcus aureus infection requiring aggressive fluid resuscitation and vasoactive support in the first $24 \mathrm{~h}$. The next day, her hemodynamics have improved, and she is able to be weaned off inotropic support, though anasarca is noted on physical exam and pulmonary edema is seen on chest radiography. An attending physician who is rounding suggests initiation of diuresis with furosemide, but another attending is concerned that aggressive medical diuresis may worsen the patient's hemodynamic status and instead recommends initiation of renal replacement therapy, specifically continuous veno-venous hemodiafiltration. A resident asks if any method of fluid removal has been proven to result better outcomes in critically ill children. 
Currently, options for managing fluid overload are limited to fluid restriction, medical management with diuretics, and/or renal replacement therapy (RRT). The decision regarding which to initiate in the setting of fluid overload is often influenced by multiple extrinsic factors including physician preference, ease of access, size of the patient, hemodynamic stability, and bleeding risk.

\section{Diuretic Therapy}

In fluid overload, diuretics are used to maximize renal salt and water excretion in the setting of volume overload. Diuretics are also used in acute renal failure with the intention of converting oliguric to non-oliguric acute renal failure. Nonoliguric renal failure has lower associated morbidity and may prevent the need for future dialysis [11]. However, the use of diuretics is associated with certain risks including electrolyte derangements, ototoxicity among the neonatal population, and AKI especially when used in conjunction with radiocontrast agents.

In patients with AKI, the effect of loop diuretics may be blunted secondary to reduced tubular secretion [12]. Therefore, it has been hypothesized that a lack of diuretic responsiveness may correlate with the degree of acute kidney injury. Kakajiwala et al. retrospectively evaluated infants after congenital heart surgery and found that responsiveness to furosemide was lower among patients with AKI. Their study defined lack of furosemide responsiveness as urine output of less than $1.7 \mathrm{~mL} / \mathrm{kg} / \mathrm{h}$ at $2 \mathrm{~h}$ or $1.9 \mathrm{~mL} / \mathrm{kg} / \mathrm{h}$ at $6 \mathrm{~h}$ after diuretic administration. After correcting for fluid balance, it was found that lack of furosemide responsiveness was predictive of AKI. While diuretic use may be beneficial in the management of fluid overload, a lack of responsiveness to diuretic therapy may be reflective of concurrent renal injury which can complicate clinical course and management.

At the moment, diuretics remain a mainstay of therapy given that worsening fluid overload contributes to increased mortality and morbidity, but the ideal method of administration remains unclear. In 1992, Singh et al. evaluated in a pro- spective randomized controlled trial continuous versus intermittent furosemide in postoperative pediatric cardiac patients. Patients were randomized to either IV intermittent $(1 \mathrm{mg} / \mathrm{kg}$ furosemide every $4 \mathrm{~h}$ to be increased by $0.25 \mathrm{mg} / \mathrm{kg}$ every $4 \mathrm{~h}$ to a maximum of $1.5 \mathrm{mg} / \mathrm{kg}$ ) or continuous IV infusion $(0.1 \mathrm{mg} / \mathrm{kg} / \mathrm{h}$ of furosemide doubled every $2 \mathrm{~h}$ to a maximum of $0.4 \mathrm{mg} / \mathrm{kg} / \mathrm{h}$ ) to maintain urine output $>1.0 \mathrm{~mL} / \mathrm{kg} / \mathrm{h}$. Data showed that those who received the continuous infusion had less variability in urine output and required a lower cumulative daily dose of furosemide, suggesting that continuous infusions may be more advantageous in this patient population [13]. Klinge et al. attempted to replicate this evaluation with 57 postoperative cardiac patients in a prospective, randomized study. Patients were given either intermittent IV furosemide or a continuous infusion of furosemide when urine output fell to less than $1 \mathrm{~mL} / \mathrm{kg} / \mathrm{h}$ and CVP was more than $5 \mathrm{cmH}_{2} \mathrm{O}$. Patients in the intermittent IV furosemide group required less furosemide over the 3-day study period ( $1.2 \mathrm{mg} / \mathrm{kg} /$ day vs $1.8 \mathrm{mg} /$ $\mathrm{kg} /$ day) to achieve the same, targeted urine volume of $>1 \mathrm{~mL} / \mathrm{kg} / \mathrm{h}$ [14]. Continuous infusions of furosemide result in more consistent hourly urine output and may be preferred to intermittent dosing in hemodynamically unstable patients.

Alternative diuretic choices to furosemide are another area of ongoing study among the pediatric population. Ethacrynic acid, which can be used as an alternative to other loop diuretics in the setting of sulfa drug allergies, has been studied dating as early as the 1960s. The mechanism of action is identical to furosemide and other loop diuretics - direct reversible blockade of $\mathrm{Na} / \mathrm{K} / 2 \mathrm{Cl}$ binding sites in the thick ascending loop of Henle. Ethacrynic acid has been reported to be $30 \%$ less potent than furosemide [15]. Ethacrynic acid was studied in 22 children aged 2-17 years of age with congestive heart failure. They reported 3.6\% average weight loss in patients administered with ten intermittent doses of ethacrynic acid, with an increase in urinary volume by a factor of 2.5 over a 24-h period and with minimal electrolyte abnormalities [16]. In 2015, Ricci et al. performed a prospective randomized double-blinded study on 74 pediatric patients undergoing elective cardiac 
surgery comparing furosemide versus ethacrynic acid. Patients were included in the study if they had clinical signs of fluid overload and randomized to either receive furosemide or ethacrynic acid, with primary outcome measuring mean urine output on postoperative day 0 . Urine output was noted to be higher among patients who received ethacrynic acid $(6.9 \mathrm{~mL} / \mathrm{kg} / \mathrm{h})$ compared with furosemide $(4.6 \mathrm{ml} / \mathrm{kg} / \mathrm{h}, \mathrm{p}=0.002)$ despite a lower cumulative dose of ethacrynic acid $(0.22 \mathrm{mg} / \mathrm{kg} / \mathrm{h}$ vs $0.33 \mathrm{mg} / \mathrm{kg} / \mathrm{h}, p<0.0001)$, suggesting that less ethacrynic acid may be required to achieve the same urine output as furosemide in this patient population. The occurrence of AKI was not statistically significant between groups, and no complications linked to electrolyte disorders were observed in either group. Based on these data, it can be concluded that ethacrynic acid is at least as efficacious as furosemide in children recovering from cardiac surgery and may have a role in other critically ill patient populations such as those with sulfa allergies. It is important to note however that ethacrynic acid is currently markedly more expensive than furosemide which, in the current health-care climate, will be a barrier to its use.

Additional diuretic options include bumetanide (a loop diuretic) and thiazide diuretics such as chlorothiazide and metolazone, which act to inhibit $\mathrm{Na} / \mathrm{Cl}$ co-transporter channels in the proximal part of the distal convoluted tubule. This latter class of diuretics, when used in conjunction with a loop diuretic, may provide a synergistic effect, given that the mechanisms of action of the two classes of drugs are different. Serum electrolytes, especially sodium, potassium, and chloride, should be monitored frequently, as the risk of electrolyte abnormalities increases when these two classes of drugs are used concurrently [17].

Aminophylline has been described as adjunctive therapy for diuresis. Aminophylline is a methylxanthine that functions as a diuretic by acting as an adenosine receptor antagonist, which serves to increase renal blood flow and inhibit solute reabsorption. In cases of oliguria refractory to traditional loop diuretics, there is some thought that aminophylline may help increase efficacy of loop diuretics by promoting dilation of afferent glomerular arterioles and increasing glomerular filtration rate (GFR) [18]. Pediatric and neonatal studies confirm benefits of adjunctive therapy of aminophylline when used in combination with furosemide therapy. Pretzlaff et al. administered a bolus of $6 \mathrm{mg} / \mathrm{kg}$ of aminophylline to pediatric patients aged 2-46 months of age with fluid overload who were concurrently treated with furosemide infusions of $\geq 6 \mathrm{mg} / \mathrm{kg} /$ day. Theophylline levels were measured as peak levels $30 \mathrm{~min}$ after administration. The mean peak level was $8.3 \mathrm{mcg} /$ $\mathrm{mL}$, and urine output was positively correlated with theophylline level. After administration, patients were noted to have a significant increase in urine output (>80\%) during the first $2 \mathrm{~h} \mathrm{[19].} \mathrm{A}$ neonatal case study described five infants, 26-38 weeks of age, receiving furosemide infusions at $0.2-0.6 \mathrm{mg} / \mathrm{kg} / \mathrm{h}$ with ongoing oliguria who were loaded with $4 \mathrm{mg} / \mathrm{kg}$ of aminophylline and treated with increasing doses until diuresis was achieved. All infants demonstrated weight loss, reduction of mean airway pressure, and $\mathrm{FiO} 2$ requirements, along with the resolution of pleural effusions. Aminophylline may therefore be helpful for patients with ongoing fluid overload despite loop diuretic therapy. For patients who receive multiple doses of aminophylline in this setting, monitoring of serum concentrations based on traditional protocols to prevent toxicity would be prudent.

\section{Renal Replacement Therapy}

The utility and timing of RRT for fluid overload remain controversial. The majority of studies that have evaluated the use of RRT as a therapeutic modality in critically ill children have done so in the setting of an underlying diagnosis of acute kidney injury and other concurrent comorbidities. Fluid overload frequently occurs in conjunction with renal disease associated with multiorgan dysfunction and is intimately intertwined physiologically with acute kidney injury. RRT for fluid overload within the critical care environment is initiated in the setting of AKI or ARF in approximately $4-8 \%$ of all intensive care patients [20-22]. 
Although there are multiple modalities of RRT available (e.g., peritoneal dialysis, intermittent hemodialysis, or continuous hemofiltration), use of continuous veno-venous hemofiltration (CVVH) or continuous veno-venous hemodiafiltration (CVVHDF) has become increasingly preferred by pediatric critical care providers [1]. Implementation of RRT for critically ill children who become anuric or develop accepted criteria for dialysis (i.e., life-threatening hyperkalemia or uremia) secondary to AKI is the standard of care and, accordingly, not controversial. Use of RRT, however, in the setting of fluid overload in patients without anuria or other definite criteria for dialysis is more debatable. Studies in pediatric patients have shown that the percentage of fluid overload prior to initiation of therapy is independently associated with survival in all patients receiving CVVH or CVVHDF [1, 20, 23]. For example, in one retrospective study of critically ill children with multiorgan dysfunction, fluid overload was independently associated with mortality; fluid overload was $15.5 \%$ in non-survivors versus $9.2 \%$ in survivors $(p<0.01)[1]$. Adult studies have shown similar findings. In one prospective study of adult patients, 90-day mortality was doubled among those who had fluid overload at the time of RRT initiation (identified as greater than $10 \%$ fluid overload) [20]. Another adult study demonstrated that increased positive mean daily fluid balance after RRT initiation was also associated with increased mortality [23]. Despite these studies and others on the use of RRT with AKI and fluid overload, the ideal timing for initiation of RRT therapies remains unclear. Perhaps more importantly, it has not been clearly demonstrated that RRT utilization for fluid overload leads to improvement in survival outcomes. A meta-analysis in 2008 evaluating all randomized controlled trials available in adult literature regarding the utilization of RRT demonstrated a mortality risk reduction that was inadequately powered to demonstrate statistical significance [24]. A similar systematic review of adult data performed in 2011 looked to compare early versus late initiation of RRT and showed an association with reduced mortality, but results were complicated by significant statistical heterogeneity [25].
In summary, fluid overload greater than $10 \%$ has been associated with increased mortality and poor outcomes in pediatric and adult patients with critical illness. Diuretic medications, with furosemide generally accepted as the first-line agent, can be beneficial in improving urine output. Method of administration (continuous vs intermittent IV) can be based on provider preference and patient factors (e.g., hemodynamic stability, available intravenous access sites, etc.). Adjunctive treatment with aminophylline may be considered in refractory oliguria in patients already receiving traditional diuretic therapies. While current literature does not support RRT implementation based solely on the percentage of fluid overload, it should be considered as therapeutic option for fluid removal in patients with persistent or worsening fluid overload greater than $10 \%$ of pre-illness weight despite maximal medical therapy. As the use of CVVH and CVVHDF becomes more commonplace, improved research will hopefully allow for better developed algorithms to dictate fluid removal therapies in the critical care setting.

\section{Fluid Overload and Mechanical Ventilation}

Case

A 4-month-old child presents to the hospital with respiratory distress in the setting of enterovirus-induced pneumonitis. At the time of initial presentation, he is noted to be tachycardic with poor urine output for which he receives aggressive fluid resuscitation. His respiratory status continues to worsen, and by day 4 of illness, he is endotracheally intubated and placed on mechanical ventilation. His chest X-ray shows increased interstitial markings consistent with pulmonary edema. The resident asks if the fluid resuscitation contributed to his decompensation and asks if this will significantly impact his recovery. 
Fluid overload and respiratory compromise are often connected, particularly in conditions such as sepsis in which systemic inflammation and endothelial injury lead to significant capillary leak and extravasation of fluid into the interstitial space. In recent years, an emphasis on increased early fluid resuscitation for septic shock and the emergence of sepsis protocols with early goal-directed therapy have created the potential for patients to experience more clinically significant long-term fluid overload.

Stabilizing hemodynamics with aggressive fluid resuscitation may come at a cost. Emerging literature shows that prolonged positive fluid balance after hemodynamic stability has been achieved can have a negative impact on organ function and respiratory recovery. These findings are present even when correcting for the underlying severity of illness at the time of presentation. For example, fluid overload $\geq 15 \%$ has been independently associated with prolonged ventilation and increased hospital length of stay [26].

Fluid overload has been associated with increased mortality and duration of mechanical ventilation in children identified with acute lung injury (ALI), [27] a finding which was also observed in the pediatric arm of the Calfactant in Acute Respiratory Distress Syndrome trial [28]. This trial was a multicenter prospective evaluation of intratracheally administered surfactant use in adult and pediatric patients diagnosed with ARDS/ALI. The pediatric arm consisted of 24 children's hospitals across 6 countries and evaluated all-cause mortality at 90 days after study entry, with secondary outcomes of oxygenation changes after intervention, ventilator-free days at 28 days, and total duration of ICU stay [29]. Greater accumulated positive fluid balance from day 1 to day 7 of illness was associated with inhospital mortality $(p<0.001)$, with non-survivors averaging $8.7 \pm 9.5 \mathrm{~L} / \mathrm{m}^{2}$ compared with $1.2 \pm 2.4 \mathrm{~L} / \mathrm{m}^{2}$ in survivors. Increased cumulative fluid overload was also associated significantly with less ventilator-free days and longer durations of PICU and hospital stay [28].

In one of the few studies that has examined fluid overload in the pediatric setting in the absence of RRT, Arikan and colleagues found that fluid overload was independently associated with compromised respiratory function. Specifically, peak fluid overload correlated significantly with peak oxygenation index (OI) and Pediatric Logistic Organ Dysfunction (PELOD) score [26]. In a single-center retrospective review of 636 mechanically ventilated children, percent fluid overload correlated with number of invasive ventilation days and $\mathrm{OI}$ at $48 \mathrm{~h}$ of mechanical ventilation [30]. There are a small number of studies that did not identify a relationship between fluid overload and respiratory morbidity. One such example is a post hoc analysis of a study conducted by the Pediatric Acute Lung Injury and Sepsis Investigators (PALISI) that did not show an association between cumulative fluid balance and other respiratory measures such as duration of ventilator weaning and extubation outcomes, though this analysis included all mechanically ventilated patients rather than the subset with ALI or ARDS [31].

While there may be some conflicting data on the relationship between fluid overload and respiratory morbidity, the overwhelming majority of published studies examining this relationship has identified negative consequences of this relationship. For this reason, some authors have hypothesized that active avoidance of early fluid overload may be an appropriate therapeutic goal for patients with underlying critical respiratory illness to reduce time on mechanical ventilation [32]. In a prospective randomized adult trial comparing conservative (i.e., maintenance of central venous pressure $\leq 4 \mathrm{mmHg}$ ) versus liberal (i.e., maintenance of central venous pressure 10-14 $\mathrm{mmHg}$ ) fluid strategies in patients with acute lung injury, oxygenation index and lung injury score were lower, and the number of ventilator free days was higher in patients who received the conservative fluid strategy. There was no statistical difference in the primary endpoint of 60-day mortality: $25.5 \%$ versus $28.4 \%$ in the conservative and liberal arms of the study, respectively [33]. To our knowledge, similar pediatric studies have not yet been conducted.

Outcomes for patients requiring mechanical ventilation are dependent on multiple factors, 
and there are enough published data to conclude that fluid overload likely contributes to worse outcomes in these patients. Moreover, there are no studies that have demonstrated any benefit of fluid overload on respiratory function or outcomes. Recent reviews of ALI and ARDS management in pediatric patients have yet to make recommendations regarding concurrent fluid management strategies [34]. Despite the current lack of guidelines, it is reasonable to conclude that careful attention to fluid management and avoidance of fluid overload whenever possible in patients with respiratory disease are warranted.

\section{Fluid Overload and Cardiac Surgery}

\section{Case \\ A 4-month-old female undergoes surgical repair of tetralogy of Fallot. She returns from the operating room endotracheally intubated on mechanical ventilation. Her first night of hospitalization is complicated by significant hemodynamic instability necessitating frequent fluid boluses. Over the next day, she becomes progressively more fluid overloaded with diffuse ana- sarca and worsening pulmonary edema. The resident asks if there are specific risks of fluid overload in the postoperative car- diac patient when compared with other patients.}

Pediatric patients undergoing cardiac surgery represent a unique population in which their pathophysiology predisposes them to fluid accumulation in the postoperative period. This predisposition can vary based on many factors including the age of the patient and the underlying comorbidities that may be present. Neonates are at particular risk to develop fluid overload due to their increased total body water distribution and renal immaturity. Indeed, fluid overload can occur early in the postoperative period, tending to develop more readily in patients that are younger with cyanotic heart lesions and/or worse kidney function at baseline [35]. Many of the children with these risk factors also have complex lesions requiring longer $\mathrm{CPB}$ durations. Prolonged exposure to CPB and the stress of surgery augments the postoperative inflammatory response and consequent capillary leak, likelihood of low cardiac output, and AKI, making fluid overload almost unavoidable in some cases. In a recent retrospective review of 193 children who underwent cardiac surgery, the amount of fluid administered in the first $6 \mathrm{~h}$ of ICU admission after surgery was the strongest independent risk factor for fluid overload by postoperative day 2 [36]. In one of the largest prospective studies to date focused on this issue, which examined characteristics and outcomes of 1525 children who underwent cardiac surgery over a 4-year period at a single institution, Lex et al. noted that patients who were more likely to develop fluid overload greater than $5 \%$ were significantly younger (median 151 days versus 448 days) and smaller $(4.3 \mathrm{~kg}$ versus $8.4 \mathrm{~kg}$ ) and had longer duration of CPB (131 min versus $78 \mathrm{~min}$ ) and aortic cross clamp (69 min versus $39 \mathrm{~min}$ ) and lower urine output on postoperative day $1(1.7 \mathrm{~mL} / \mathrm{kg} / \mathrm{h}$ versus $2.6 \mathrm{~mL} / \mathrm{kg} / \mathrm{h})$ [37]. A summary of all potential risk factors for fluid overload is listed in Table 11.2.

Multiple studies have found associations of fluid overload with worse outcomes after pediatric cardiac surgery [38]. For instance, a retro-

Table 11.2 Risk factors for fluid overload in cardiac surgery

\begin{tabular}{l}
\hline Preoperative \\
\hline$\bullet$ Fluid overload secondary to heart failure \\
\hline - Diminished baseline renal function \\
\hline - Younger age \\
\hline - Lower body weight \\
\hline - Cyanotic congenital heart disease \\
\hline Intraoperative \\
\hline Excessive exogenous fluid and blood products for \\
during surgery \\
\hline - Longer duration of cardiopulmonary bypass and \\
aortic cross clamp \\
\hline Postoperative \\
\hline - Acute kidney injury \\
• Hemodynamic instability requiring aggressive \\
fluid resuscitation
\end{tabular}


spective cohort study evaluating 435 neonates undergoing cardiac surgery also recognized this relationship, with fluid overload $\geq 16 \%$ in the postoperative period that was independently associated with worse outcomes including longer hospital stay. These patients were also more likely to experience cardiac arrest requiring CPR, develop thrombosis, or require chest re-exploration. Hazle et al. prospectively identified fluid overload within the first 3 postoperative days following cardiac surgery to be independently associated with poor outcomes in 49 infants $<6$ months of age who underwent surgery with CPB [38]. Specifically, they found that odds of poor outcome (defined by a composite score that included the need for RRT, time to first extubation, intensive care length of stay, or death within 30 days of surgery) increased by $7 \%$ with each $1 \%$ increase in fluid overload as calculated by maximum daily weight method, adjusted for peak serum creatinine [38].

Fluid overload is therefore deemed to be hazardous to the postoperative pediatric cardiac patient by current pediatric cardiac intensive care providers. As a result, many centers implement management strategies that are designed to prevent fluid overload. These measures include some or all of the following: intraoperative and postoperative corticosteroids to reduce immunogenic-mediated capillary leak, conservative fluid management, early diuretic administration to mitigate perioperative fluid/blood product administration, and dialysis. Emerging evidence suggests that early dialysis, particularly with peritoneal dialysis (PD), may have benefits to postoperative cardiac patients [39]. A single-center retrospective study of 146 neonates published in 2012 reported that children with AKI after cardiac surgery in which PD was started early (within the first day) had lower 30- and 90-day mortality rates [40]. Of note, 52 complications related to PD were recorded in 44 patients in this study including dysfunction of the catheter (leakage at the insertion site, displacement, or insufficient drainage), hydrothorax, hemoperitoneum related to insertion or withdrawal of the catheter, bowel perforation, and peritonitis. More recently, in an unblinded trial by Kwiatkowski et al., 73 infants with postoperative oliguria (defined as 4 total h of urine output $<1 \mathrm{~mL} / \mathrm{kg} / \mathrm{h}$ during the first postoperative $24 \mathrm{~h}$ ) were randomized to receive either PD or furosemide therapy. Patients who were restricted to furosemide treatment were more likely to develop $10 \%$ fluid overload, prolonged mechanical ventilation, and prolonged CICU stay. Also, patients who received PD had fewer electrolyte abnormalities, though two PD-related adverse outcomes were observed peritoneal bleeding requiring a blood transfusion and hydrocele formation [39].

In summary, postoperative cardiac patients are predisposed to fluid overload due to a variety of factors. Significant fluid overload in postoperative cardiac patients is common and associated with worse outcomes. While fluid overload may be unavoidable in some patients, efforts to minimize the degree of fluid overload are appropriate. For institutions that have the resources and experience to readily implement PD in the early postoperative period, careful risk factor assessment could drive the creation of PD protocols for this patient population. As experience with this modality increases and more data become available, many of the risks associated with PD may decrease, and centers may be more inclined to offer this modality more frequently. In the meantime, conservative fluid management, early initiation of diuretic therapy, and careful attention to daily fluid balance and weight measurements are necessary to temper the risks of fluid overload in children recovering from cardiac surgery.

\section{Fluid Overload and ECMO}

\section{Case}

A 6-month-old infant with a history of prematurity and chronic lung disease presents to the hospital in respiratory distress from respiratory syncytial virus infection. She is endotracheally intubated and provided mechanical ventilation for respiratory failure with hypoxemia. Despite multiple ventilator maneuvers, her arterial blood gases 
show worsening, severe hypercarbia. She becomes increasingly acidotic and develops hemodynamic instability necessitating aggressive fluid resuscitation. Shortly thereafter, she has a cardiac arrest and is cannulated emergently for venoarterial extracorporeal membrane oxygenation (ECMO). After cannulation, she is noted to be increasingly edematous, which is distressing for the family. They ask if her edema has any implications on her clinical outcome.

In recent years, extracorporeal membrane oxygenation (ECMO) has become a more readily accessible resource for the management of critically ill children, with the number of ECMO centers increasing on a yearly basis [41]. AKI is a common comorbidity for children undergoing ECMO support and has been associated with increased mortality [42]. The burden of illness suffered by children requiring ECMO support makes them prone to more aggressive fluid resuscitation and, accordingly, more fluid accumulation. Despite concerns that fluid overload may be harmful in a variety of clinical situations including mechanical ventilation and postoperative care, there are few studies evaluating its effect, independent of AKI, on patients receiving ECMO.

Of the limited published data, fluid overload on ECMO has been associated with adverse outcomes in neonatal, pediatric, and adult patients. Neonatal studies of children with pulmonary hypertension on ECMO have suggested that edema and fluid mobilization play a role in persistently poor respiratory function and duration of ECMO [43, 44]. Selewski and colleagues retrospectively evaluated fluid overload in 756 pediatric ECMO courses at 6 centers within the United States and Canada. In this study, patients who died while on ECMO had significantly greater peak fluid overload while on ECMO, greater change in fluid overload during their ECMO course, and greater fluid overload at time of ECMO discontinuation. More importantly, in a multivariable analysis, peak fluid overload while on ECMO independently predicted mortality (adjusted odds ratio, 1.09; 95\% CI, 1.041.15); and both fluid overload at ECMO initiation (adjusted odds ratio, 1.13; 95\% CI, 1.05-1.22) and peak fluid overload (adjusted odds ratio, 1.18 ; $95 \%$ CI, 1.12-1.24) predicted hospital mortality, independent of AKI [45]. Though the study population was largely neonates and the diagnosis of AKI was made using serum creatinine values, these multicenter data highlight a potential effect of fluid overload on morbidity and mortality.

Based on current literature, it is unclear whether conservative fluid management, diuretic therapy, or RRT, either alone or in some combination, is a superior management strategy for patients on ECMO. Current CRRT devices can easily be utilized in conjunction with the ECMO circuit, eliminating access as a barrier to its use in this patient population. As a result, many centers have a low threshold for initiating CRRT for ECMO patients. Studies have shown that neonatal ECMO survivors receiving CRRT had an overall better fluid balance and received less diuretic therapy compared with non-CRRT survivors [46]. A systematic review performed of all published literature regarding CRRT and ECMO showed that ECMO survivors receiving CRRT therapy had an overall fluid balance that was less positive compared with those not on CRRT but also noted studies showing longer ECMO duration when CRRT was applied [47]. Additionally, other studies have shown an increased association between mortality and concomitant CRRT on ECMO [48, 49]. Unfortunately, it is unlikely that a prospective RCT will be conducted on the use of CRRT in ECMO patients, as there is no consensus on accepted indications for initiation and practice varies considerably across centers. The risk/benefit ratio must be evaluated for each patient accounting for resource availability, nutritional needs, and the potential for worsening intravascular volume depletion, which can further exacerbate AKI and negatively affect the function of the ECMO circuit. 


\section{Conclusion}

Fluid overload remains an area of ongoing evolution regarding its definition and management within the clinical setting of the critically ill child. Despite a large body of evidence to indicate that it is associated with increased morbidity and mortality in specific disease states, management strategies are largely dictated by physician experience and preference. Importantly, it can be stated with high confidence that there are no studies to date that suggest that fluid overload is beneficial to patients or has any positive effect on recovery or survival. Definitions of fluid overload have largely relied upon calculated measurements, but these may underrepresent fluid distribution during critical illness and fail to completely capture the intricacies of fluid extravasation that put certain organs at higher risk of complications than others. Clinicians should focus on judicious use of fluids including blood products along with careful assessment of fluid status, thereby allowing for early interventions aimed at prevention of fluid overload states and the associated cellular edema, organ dysfunction, morbidity, and mortality.

\section{Key Points}

- The dynamics of fluid movement are complex, and our understanding regarding such is still evolving.

- Current methods of the assessment of fluid overload are potentially error prone and can produce conflicting results. Daily assessment of fluid status however is crucial, and discrepancies between weight and intake/output calculations should be reconciled by clinical exam and available physiologic bedside data.

- Fluid overload is associated with adverse outcomes in multiple disease states, and, to our knowledge, there are no studies that demonstrate that fluid overload is beneficial to patients or their outcomes.
- Options for management of fluid overload include conservative fluid management, diuretic therapy, and RRT including CVVH/CVVHDF and PD. Furosemide therapy is generally accepted as the first-line diuretic agent, but persistent or worsening fluid overload despite conservative fluid management and furosemide therapy should prompt adjunctive treatments or RRT.

- Further research of the effects of fluid overload and its management in specific patient populations and disease states is required to improve our practice and decrease variations in care across centers.

\section{References}

1. Foland JA, Fortenberry JD, Warshaw BL, Pettignano R, Merrittv RK, Heard ML, Rogers K, Reid C, Tanner AJ, Easley KA. Fluid overload before csontinuous hemofiltration and survival in critically ill children: a retrospective analysis. Crit Care Med. 2004;32:1771-6.

2. Jain A. Body fluid composition. Pediatr Rev. 2015;36:141-52.

3. Bianchetti MG, Simonetti GD, Bettinelli A. Body fluids and salt metabolism - part I. Ital J Pediatr. 2009;35:36.

4. Agrò F.E. VM. Physiology of body fluid compartments and body fluid movements. Milano: Springer; 2013.

5. Woodcock TE, Woodcock TM. Revised Starling equation and the glycocalyx model of transvascular fluid exchange: an improved paradigm for prescribing intravenous fluid therapy. Br J Anaesth. 2012;108:384-94.

6. Rogers' Textbook of Pediatric Intensive Care. 5th ed. Philadelphia: Wolters Kluwer; 2008.

7. Holte K, Sharrock NE, Kehlet H. Pathophysiology and clinical implications of perioperative fluid excess. Br J Anaesth. 2002;89:622-32.

8. Selewski DT, Cornell TT, Lombel RM, Blatt NB, Han YY, Mottes T, Kommareddi M, Kershaw DB, Shanley TP, Heung M. Weight-based determination of fluid overload status and mortality in pediatric intensive care unit patients requiring continuous renal replacement therapy. Intensive Care Med. 2011;37:1166-73.

9. Goldstein SL, Currier H, Graf C, Cosio CC, Brewer ED, Sachdeva R. Outcome in children receiving continuous venovenous hemofiltration. Pediatrics. 2001;107:1309-12. 
10. Milani GP, Groothoff JW, Vianello FA, Fossali EF, Paglialonga F, Edefonti A, Agostoni C, Consonni D, van Harskamp D, van Goudoever JB, Schierbeek H, Oosterveld MJS. Bioimpedance and fluid status in children and adolescents treated with Dialysis. Am J Kidney Dis. 2017;69:428-35.

11. Mehta RL, Pascual MT, Soroko S, Chertow GM, for the PSG. Diuretics, mortality, and nonrecovery of renal function in acute renal failure. JAMA. 2002;288:2547-53.

12. Kakajiwala A, Kim JY, Hughes JZ, Costarino A, Ferguson J, Gaynor JW, Furth SL, Blinder JJ. Lack of furosemide responsiveness predicts acute kidney injury in infants after cardiac surgery. Ann Thorac Surg. 2017;104:1388-94.

13. Singh NC, Kissoon N, al Mofada S, Bennett M, Bohn DJ. Comparison of continuous versus intermittent furosemide administration in postoperative pediatric cardiac patients. Crit Care Med. 1992;20:17-21.

14. Klinge JM, Scharf J, Hofbeck M, Gerling S, Bonakdar $\mathrm{S}$, Singer H. Intermittent administration of furosemide versus continuous infusion in the postoperative management of children following open heart surgery. Intensive Care Med. 1997;23:693-7.

15. Ricci Z, Haiberger R, Pezzella C, Garisto C, Favia I, Cogo P. Furosemide versus ethacrynic acid in pediatric patients undergoing cardiac surgery: a randomized controlled trial. Crit Care. 2015;19:2.

16. Sparrow AW, Friedberg DZ, Nadas AS. The use of ethacrynic acid in infants and children with congestive heart failure. Pediatrics. 1968;42:291-302.

17. Roush GC, Kaur R, Ernst ME. Diuretics: a review and update. J Cardiovasc Pharmacol Ther. 2013;19:5-13.

18. Ng GYT, Baker EH, Farrer KFM. Aminophylline as an adjunct diuretic for neonates-a case series. Pediatr Nephrol. 2005;20:220-2.

19. Pretzlaff RK, Vardis RJ, Pollack MM. Aminophylline in the treatment of fluid overload. Crit Care Med. 1999;27:2782-5.

20. Vaara ST, Korhonen A-M, Kaukonen K-M, Nisula $\mathrm{S}$, Inkinen $\mathrm{O}$, Hoppu S, Laurila JJ, Mildh L, Reinikainen M, Lund V. Fluid overload is associated with an increased risk for 90-day mortality in critically ill patients with renal replacement therapy: data from the prospective FINNAKI study. Crit Care. 2012;16:R197.

21. Uchino S, Kellum JA, Bellomo R, Doig GS, Morimatsu H, Morgera S, Schetz M, Tan I, Bouman C, Macedo E, Gibney N, Tolwani A, Ronco C. Acute renal failure in critically ill patients: a multinational, multicenter study. JAMA. 2005;294:813-8.

22. Bagshaw SM, Laupland KB, Doig CJ, Mortis G, Fick GH, Mucenski M, Godinez-Luna T, Svenson LW, Rosenal T. Prognosis for long-term survival and renal recovery in critically ill patients with severe acute renal failure: a population-based study. Criti Care. 2005;9:R700-9.

23. Bellomo R, Cass A, Cole L, Finfer S, Gallagher M, Lee J, Lo S, McArthur C, McGuiness S, Norton R, Myburgh J, Scheinkestel C, Su S. An observa- tional study fluid balance and patient outcomes in the randomized evaluation of normal vs. augmented level of replacement therapy trial. Crit Care Med. 2012;40:1753-60.

24. Seabra VF, Balk EM, Liangos O, Sosa MA, Cendoroglo M, Jaber BL. Timing of renal replacement therapy initiation in acute renal failure: a metaanalysis. Am J Kidney Dis. 2008;52:272-84.

25. Karvellas CJ, Farhat MR, Sajjad I, Mogensen SS, Leung AA, Wald R, Bagshaw SM. A comparison of early versus late initiation of renal replacement therapy in critically ill patients with acute kidney injury: a systematic review and meta-analysis. Criti Care. 2011;15:R72.

26. Arikan AA, Zappitelli M, Goldstein SL, Naipaul A, Jefferson LS, Loftis LL. Fluid overload is associated with impaired oxygenation and morbidity in critically ill children. Pediatr Crit Care Med. 2012;13:253-8.

27. Flori HRCG, Liu KD, et al. Positive fluid balance is associated with higher mortality and prolonged mechanical ventilation in pediatric patients with acute lung injury. Crit Care Res Pract. 2011;854142

28. Willson DF, Thomas NJ, Tamburro R, Truemper E, Truwit J, Conaway M, Traul C, Egan EE. The relationship of fluid administration to outcome in the pediatric calfactant in acute respiratory distress syndrome trial. Pediatr Crit Care Med. 2013;14:666-72.

29. Willson DF, Thomas NJ, Tamburro R, Truemper E, Truwit J, Conaway M, Traul C, Egan EE. Pediatric calfactant in acute respiratory distress syndrome trial. Pediatr Crit Care Med. 2013;14:657-65.

30. Sinitsky L, Walls D, Nadel S, Inwald DP. Fluid overload at 48 hours is associated with respiratory morbidity but not mortality in a general PICU: retrospective cohort study. Pediatr Crit Care Med. 2015;16:205-9.

31. Randolph AG, Forbes PW, Gedeit RG, Arnold JH, Wetzel RC, Luckett PM, O'Neil ME, Venkataraman ST, Meert KL, Cheifetz IM, Cox PN, Hanson $\mathrm{JH}$. Cumulative fluid intake minus output is not associated with ventilator weaning duration or extubation outcomes in children. Pediatr Crit Care Med. 2005;6:642-7.

32. Ingelse SA, Wiegers HM, Calis JC, van Woensel JB, Bem RA. Early fluid overload prolongs mechanical ventilation in children with viral-lower respiratory tract disease. Pediatr Crit Care Med. 2017;18:e106-11.

33. Wiedemann HP, et al. Comparison of two fluid-management strategies in acute lung injury. N Engl J Med. 2006;354(24):2564-75.

34. Randolph AG. Management of acute lung injury and acute respiratory distress syndrome in children. Crit Care Med. 2009;37:2448-54.

35. Hassinger AB, Wald EL, Goodman DM. Early postoperative fluid overload precedes acute kidney injury and is associated with higher morbidity in pediatric cardiac surgery patients. Pediatr Crit Care Med. 2014;15:131-8.

36. Seguin J, Albright B, Vertullo L, Lai P, Dancea A, Bernier P-L, Tchervenkov CI, Calaritis C, Drullinsky D, Gottesman R. Extent, risk factors, and outcome of 
fluid overload after pediatric heart surgery. Crit Care Med. 2014;42:2591-9.

37. Lex DJ, Toth R, Czobor NR, Alexander SI, Breuer T, Sapi E, Szatmari A, Szekely E, Gal J, Szekely A. Fluid overload is associated with higher mortality and morbidity in pediatric patients undergoing cardiac surgery. Pediatr Crit Care Med. 2016;17:307-14.

38. Hazle MA, Gajarski RJ, Yu S, Donohue J, Blatt NB. Fluid overload in infants following congenital heart surgery. Pediatr Crit Care Med. 2013;14:44-9.

39. Kwiatkowski DM, Goldstein SL, Cooper DS, Nelson DP, Morales DS, Krawczeski CD. Peritoneal dialysis vs furosemide for prevention of fluid overload in infants after cardiac surgery: a randomized clinical trial. JAMA Pediatr. 2017;171:357-64.

40. Bojan M, Gioanni S, Vouhe PR, Journois D, Pouard P. Early initiation of peritoneal dialysis in neonates and infants with acute kidney injury following cardiac surgery is associated with a significant decrease in mortality. Kidney Int. 2012;82:474-81.

41. ECMO Registry of the Extracorporeal Life Support Organization (ELSO). 2017.

42. Fleming GM, Sahay R, Zappitelli M, King E, Askenazi DJ, Bridges BC, Paden ML, Selewski DT, Cooper DS. The incidence of acute kidney injury and its effect on neonatal and pediatric extracorporeal membrane oxygenation outcomes: a multicenter report from the kidney intervention during extracorporeal membrane oxygenation study group. Pediatr Crit Care Med. 2016;17:1157-69.

43. Kelly RE Jr, Phillips JD, Foglia RP, Bjerke HS, Barcliff LT, Petrus L, Hall TR. Pulmonary edema and fluid mobilization as determinants of the duration of ECMO support. J Pediatr Surg. 1991;26:1016-22.

44. Swaniker F, Kolla S, Moler F, Custer J, Grams R, Barlett R, Hirschl R. Extracorporeal life support outcome for 128 pediatric patients with respiratory failure. J Pediatr Surg. 2000;35:197-202.

45. Selewski DT, Askenazi DJ, Bridges BC, Cooper DS, Fleming GM, Paden ML, Verway M, Sahay R, King E, Zappitelli M. The impact of fluid overload on outcomes in children treated with extracorporeal membrane oxygenation: a multicenter retrospective cohort study. Pediatr Crit Care Med. 2017;18:1126-35.

46. Hoover NG, Heard M, Reid C, Wagoner S, Rogers K, Foland J, Paden ML, Fortenberry JD. Enhanced fluid management with continuous venovenous hemofiltration in pediatric respiratory failure patients receiving extracorporeal membrane oxygenation support. Intensive Care Med. 2008;34:2241-7.

47. Chen H, Yu RG, Yin NN, Zhou JX. Combination of extracorporeal membrane oxygenation and continuous renal replacement therapy in critically ill patients: a systematic review. Criti Care. 2014;18:675.

48. Paden ML, Warshaw BL, Heard ML, Fortenberry JD. Recovery of renal function and survival after continuous renal replacement therapy during extracorporeal membrane oxygenation. Pediatr Crit Care Med. 2011;12:153-8.

49. Askenazi DJ, Ambalavanan N, Hamilton K, Cutter G, Laney D, Kaslow R, Georgeson K, Barnhart DC, Dimmitt RA. Acute kidney injury and renal replacement therapy independently predict mortality in neonatal and pediatric noncardiac patients on extracorporeal membrane oxygenation. Pediatr Crit Care Med. 2011;12:e1-6. 